\title{
Energy-Efficient Opportunistic Network Coding with Congestion Control for Heterogeneous WSNs ${ }^{*}$
}

\author{
Jingwen Miao", Hang Shen ${ }^{2}$, Guangwei Bai ${ }^{1,2}$ \\ ${ }^{1}$ College of Electronics and Information Engineering, Nanjing University of Technology, Nanjing 210009, China \\ ${ }^{2}$ College of Computer Science and Engineering, Nanjing University of Science and Technology, Nanjing 210094, China \\ Corresponding author: Guangwei Bai, E-mail: bai@njut.edu.cn, Tel.\& Fax: +86-25-58139500
}

\begin{abstract}
The network coding technique is promising for improving the performance of wireless sensor networks. However, most existing network coding mechanisms omit energy or congestion metric, resulting in degradation in energy efficiency and data transmission quality. This paper proposes an Energy-efficient Opportunistic Network Coding mechanism (EONC) to efficiently deliver data in heterogeneous wireless sensor networks. We begin with a dynamic classification approach of energy and congestion levels for the nodes that participant in opportunistic forwarding, building upon which an adaptive opportunistic forwarding method is present to efficiently utilize energy and bandwidth resources. On this basis, a multi-priority data scheduling strategy, working together with the network coding mechanism, is design to ensure that packets have a better chance to be coded while satisfying requirements in energy efficiency and congestion avoidance. Our simulation results demonstrate that the EONC performs better than typical network coding mechanisms, in terms of energy utilization efficiency and communication performance.
\end{abstract}

Index Terms - Wireless Sensor Networks; Network Coding; Energy-Efficient; Congestion Contro

\section{Introduction}

Wireless sensor networks (WSNs) have been widely used to perceive and interact with the physical world ${ }^{[1]}$. WSN is a dynamic environment. Sensor nodes in WSNs have severe resource constraints including bandwidth, energy, storage, and achievable data rate. Additionally, heterogeneity ${ }^{[2]}$ is one of the characteristics of WSNs. The new nodes added to the networks will own more energy than the old ones. Even though the nodes are equipped with the same energy at the beginning, the networks cannot evolve equably for each node in expending energy, due to the radio communication characteristics, random events such as short-term link failures or morphological characteristics of the field ${ }^{[3]}$. As a result, WSN are more possibly heterogeneous networks than homogeneous ones. These characteristics identify significant challenges for providing reliable data transmission, and have drawn tremendous attention from academia and industry.

Recent studies show that network coding has great potential to improve the performance of WSNs ${ }^{[4][5][6]}$. The concept of network coding is first proposed for noiseless wired networks to achieve the multicast capacity of the underlying network graph ${ }^{[7]}$. The essential idea of network coding is to allow coding capability at network nodes (routers, relays, etc.) in exchange for capacity gain. Shortly after theoretical explorations in wired networks, the use of network coding in wireless networks towards improving throughput has been widely recognized. S. Katti et al propose the first practical network coding mechanism namely COPE ${ }^{[8]}$ for supporting efficient unicast communication in wireless network. In COPE, packet mixing can only be performed at the joint nodes of the paths determined by the routing module. This significantly limits the coding opportunities in the network. To address this problem, a MAC-layer mixing method (BEND) ${ }^{[9]}$ is proposed to proactively seize opportunities for coding. Without relying on fixed forwarders, BEND allows each node in the neighborhood to be a potential coder and forwarder and coordinates their packet transmissions for higher coding gain. However, BEND does not consider any energy or congestion metric, resulting in degradation in energy efficiency and data transmission quality.

The focus of this work is on performance improvement of energy efficiency and data communication over WSNs. In this paper, we propose an Energy-efficient Opportunistic Network Coding mechanism (EONC) to efficiently deliver data in heterogeneous WSNs, in consideration of congestion control. At first, we integrate a dynamic classification approach in accordance with energy and congestion levels of the nodes that participant in opportunistic forwarding. Then, an adaptive opportunistic forwarding method is present to achieve energyefficient and congestion adaptive data delivery. Finally, we design a multi-priority data scheduling strategy, working together with the network coding mechanism, to ensure that packets have a better chance to be coded. Our simulation results demonstrate that, compared with typical network coding mechanisms COPE and BEND, the proposed EONC achieves significant performance improvement in terms of energy efficiency and data transmission quality.

The remainder of this paper is organized as follows. Section 2 presents an investigation and understanding of existing problems of network coding over WSNs. In Section 3, we propose the EONC and present the details of its implementation. Section 4 involves thorough analysis and evaluation of the EONC performance in simulation method.

\footnotetext{
* This work is partially supported by CNSF Grant \# 60673185 and 61073197 to Guangwei Bai ,the NSF of Jiangsu Province Grant \# BK2010548 to Guangwei Bai and the Research Innovation Program for College Graduates in Jiangsu Province Grant \# CXLX11_0262 to Hang Shen
} 
Finally, Section 5 concludes this paper and outlines some perspectives for future work.

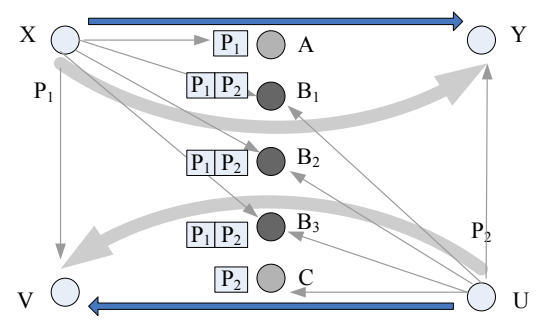

Fig. 1. Neighborhood packet repository in BEND

\section{Problem Description}

As mentioned above, existing opportunistic network coding mechanisms (such as BEND ${ }^{[9]}$ ) does not take the inherent characteristics of WSNs into account, and therefore cannot be efficiently applied in WSNs.

Consider an example of BEND in Fig. 1. Packet $p_{1}$ goes from node $\mathrm{X}$ via $\mathrm{A}$ to $\mathrm{Y}$, and another packet $p_{2}$ goes from node $\mathrm{U}$ via $\mathrm{C}$ to $\mathrm{V}$. These routes are determined by the routing protocol. By taking advantages of the broadcast nature of wireless communications, nodes $\mathrm{B}_{1}, \mathrm{~B}_{2}$ and $\mathrm{B}_{3}$ can overhear the transmissions of $p_{1}$ and $p_{2}$. Traditional methods (such as COPE) simply discard these overheard packets to avoid duplication, thus missing potential coding opportunities. Instead, BEND seizes the coding opportunities by enabling any one of $\mathrm{B}_{1}, \mathrm{~B}_{2}$ and $\mathrm{B}_{3}$ to forward the coded packet $p_{1} \oplus p_{2}$.

Although BEND can capture potential coding opportunities, it does not consider the metrics with respect to energy and congestion. In heterogeneous WSNs, we assume that two nodes capture the same coding opportunities and have the same forwarding probability. Due to energy heterogeneity, we determine that the node with lower residual energy may die more quickly, thus decreasing the network lifetime. On the other hand, the communication performance will degrade if the node with higher-level congestion forwards the packets. These existing problems motivate us to propose the EONC.

\section{Energy-Efficient Opportunistic Network Coding}

We first describe our network coding model and introduce the notations used in this section. On this basis, the main mechanisms of the EONC are comprised of the following three parts: 1) dynamic classification approach is used to classify forwarding nodes in accordance with different energy and congestion levels; 2) an adaptive opportunistic forwarding strategy is designed to efficiently utilize energy and avoid traffic congestion; 3) a multi-priority data scheduling strategy is used to achieve a tradeoff between throughput and resources utilize efficiency.

\section{A. Network Coding Model}

Consider the application of network coding in WSNs. We assume that intermediate nodes can perform network coding operations and combine packets from multiple incoming flows into a single outgoing packet. This packet is broadcasted to the entire neighborhood, thus reaching corresponding neighboring nodes. The network interface of node is set in promiscuous mode. The sensor nodes can snoop on all transmissions in their neighborhood and store the overheard data packets, whether they are intended for them or not. In this way, the sensor nodes can know the overheard and routed packets that each neighboring node possesses such that it can make network coding operations based on this information.

Let $n h_{m}$ and $p f_{m}$ be next-hop receiver and previous forwarder of packets. The set of one-hop neighbors of $p f_{m}$ is defined as $n b\left(p f_{m}\right)$. At each intermediate node, multiple packets can be mixed in a single transmission. For each pair of packets $p_{1}$ and $p_{2}$ in the set of packets to be combined, they must satisfy the following criteria:

$$
\begin{aligned}
& \text { (1) } n h_{1}=p f_{2} \vee n h_{1} \in n b\left(p f_{2}\right) \\
& \text { (2) } n h_{2}=p f_{1} \vee n h_{2} \in n b\left(p f_{1}\right)
\end{aligned}
$$

Consider an energy heterogeneous WSN environment. The sensor nodes are equipped with the different energy at the beginning. To avoid the frequent change of the topology, we assume that the nodes are micro mobile or stationary.

\section{B. Dynamic Classification Approach of Forwarding Nodes}

The energy efficiency and congestion control of WSNs are two major issues that impact the application of such networks. In EONC, each node maintains and updates a one-hop neighbor information table in real time. Each entry consists three parts, i.e. node identity, residual energy and queue length. Each node periodically detects the identities of nodes that participant in opportunistic forwarding, denoted by $M$.

Let $E\left(C_{j}\right)$ denote residual energy of node $C_{j}$. The average residual energy of nodes that belong to $M$ is given by

$$
E_{a}=\frac{1}{|M|} \sum_{j \in M} E\left(C_{j}\right)
$$

The standard deviation of residual energy of nodes belonging to $M$ is given by

$$
E_{s}=\sqrt{\frac{1}{|M|} \sum_{j \in M}\left(E\left(C_{j}\right)-E_{a}\right)^{2}}
$$

Definition 1: Energy threshold is a metric that indicate different energy level of forwarding nodes.

The energy threshold, denoted by $E_{t h}$, is defined as

$$
E_{t h}=\alpha\left(E_{a}+E_{s}\right), \alpha \in(0,1)
$$

where $\alpha$ is a parameter that can be adjusted in accordance with the changes in network environment.

Let $M_{h}$ denote the set of forwarding nodes whose residual energy is larger than $E_{t h}$.

$$
M_{h}=\left\{C_{j} \mid E\left(C_{j}\right)>E_{t h}\right\}
$$

Obviously, there always exists at least one forwarding node belonging to $M_{h}$.

Congestion control is another important issue for WSNs. Congestion not only leads to packet loss, but also increases delays and lowers network throughput with a lot of energy wastage due to retransmissions ${ }^{[10]}$. Intuitively, the buffered queue length can act as an indicator showing how busy the 
node is and the delay for incoming traffic. Once queue length reaches a certain threshold, which always means congestion. This requires us to take the queue length of nodes in forwarder list into account when classifying congestion level.

Note that we only classify the congestion level among the forwarding nodes belonging to $M_{h}$. Let $L\left(C_{j}\right)$ denote queue length of node $C_{j}$. The average queue length of nodes that belong to $M_{h}$ is given by

$$
L_{a}=\frac{1}{\left|M_{h}\right|} \sum_{j \in M_{h}} L\left(C_{j}\right)
$$

The standard deviation of queue length of nodes that belong to $M_{h}$ is given by

$$
L_{s}=\sqrt{\frac{1}{\left|M_{h}\right|} \sum_{j \in M_{h}}\left(L\left(C_{j}\right)-L_{a}\right)^{2}}
$$

Definition 2: Queue length threshold is a metric that indicate different congestion level of forwarding nodes.

The queue length threshold, denoted by $L_{t h}$, is defined as

$$
L_{t h}=\beta\left(L_{a}+L_{s}\right), \beta \geq 1
$$

where $\beta$ is a parameter that can be adjusted in accordance with the changes in network environment.

Let $N_{h}$ denote the set of forwarding nodes whose queue length is not greater than $L_{t h}$.

$$
N_{l}=\left\{C_{j} \mid L\left(C_{j}\right) \leq L_{t h}\right\}
$$

Like (4), there always exists at least one forwarding node belonging to $N_{h}$.

\section{Adaptive Opportunistic Forwarding Method}

The received and overheard data packets can be divided into three types, i.e. overhead non-coded packet (say ONCP), intended non-coded packet (say INCP) and coded Packet (say $\mathrm{CP}$ ). According to energy and congestion levels, each node can decide whether to participate in opportunistic forwarding. The method is realized in a localized way.

We define random variable $\delta$ to determine whether a node $C_{j}$ participates in opportunistic forwarding.

$$
\delta= \begin{cases}1 & j \in M_{h} \\ 0 & j \in M_{l}\end{cases}
$$

On the one hand, a node only delivers ONCPs and INCPs by opportunistic forwarding if $\delta=0$. On the other hand, all types of packets are allowed to be delivered by opportunistic forwarding if $\delta=1$.

\section{Multi-Priority Data Scheduling Strategy}

To capture potential coding opportunities, EONC adopts a multi-priority data scheduling strategy. The priority settings should take special issues resulting from WSNs into account, such as data exchange gains, energy and congestion levels, in which how to enhance data exchange gains is a major concern when performing network coding. As an opportunistic network coding mechanism, BEND [9] assigns coded transmissions higher priority, based on the number of the original packets, in packet scheduling. However, BEND does not consider any metrics about energy and congestion, and therefore cannot be efficiently applied in WSNs.

TABLE I Parameters for packet prioritization.

\begin{tabular}{|c|c|c|c|c|}
\hline Type & Classification & $C W_{\text {Min }}$ & $C W_{M a x}$ & AIFS \\
\hline ONCP & $C_{j} \in M$ & 99 & 2047 & $7 \times$ slot time + SIFS \\
\hline \multirow{2}{*}{ INCP } & $C_{j} \in N_{h}$ & 63 & 1023 & $4 \times$ slot time + SIFS \\
\cline { 2 - 5 } & $C_{j} \in N_{l}$ & 41 & 1023 & $3 \times$ slot time + SIFS \\
\hline \multirow{2}{*}{$\mathrm{CP}$} & $C_{j} \in N_{h}$ & 23 & 63 & $2 \times$ slot time + SIFS \\
\cline { 2 - 5 } & $C_{j} \in N_{l}$ & 9 & 63 & $2 \times$ slot time + SIFS \\
\hline
\end{tabular}

When the forwarder nodes contend with each other to transmit their scheduled packets, EONC prioritizes them by assigning them different back-off durations before medium access based on packet type, energy and congestion levels. It is implemented through a BEND-like type-specific method shown in Table I.

Unlike BEND, we introduce the energy and congestion levels into the assignment of packet prioritization. In this way, we can achieve a tradeoff between throughput and resources utilize efficiency.

After a node decides to send a type of packet, it must back off for a fixed period (AIFS) and another time interval uniformly distributed between 0 and $C W$, where $C W$ is a changing contention window size, to coordinate contending nodes. Initially, $C W$ is set to $C W_{\text {Min }}$ and is doubled every time a transmission attempt fails until it reaches the specified $C W_{\text {Max }}$. The smaller $C W_{\text {Min }}, C W_{\text {Max }}$ and AIFS are, the higher priority given to the packet.

\section{Performance Evaluation}

This section involves thorough performance analyses and evaluation of EONC in simulation methodology. We design and conduct a series of simulation experiments. Section A describes the evaluation metrics and detailed simulation parameter settings. Section B presents and analyses the simulation results.

\section{A. Performance Metrics}

In this work, we use network simulator $\mathrm{NS}-2^{[11]}$ to implement the proposed algorithms and compare it with typical network coding mechanisms, i.e., COPE and BEND. We now evaluate the EONC performance in a 3-tier scenario, where tiers 1, 2 and 3 each consist of 5, 8 and 5 nodes, referred to as 5-8-5 topologies, respectively. We set the separation distance between tiers so that data flows between tiers 1 and 3 must use tier-2 node(s) as forwarders.

We place CBR flows randomly between tiers 1 and 3 , two in the forward direction and two in the reverse direction. The packet transmission interval is set to be $0.01 \mathrm{~s}$. We fix the data rate at $1 \mathrm{Mbps}$, the basic rate, without any rate adaptation. To construct heterogeneous WSN environment, the sensor nodes are equipped with different energy at the beginning.

\section{B. Analysis of Simulation Results}

Figure 2 shows the throughput under different pairs of CBR flows. Here we use the number of successful transmissions to represent the throughput. It is shown that both EONC and 
BEND performs better than COPE. On the other hand, we can observe that there is a little difference between EONC and BEND. This is because EONC achieve a tradeoff between throughput and resources utilize efficiency.

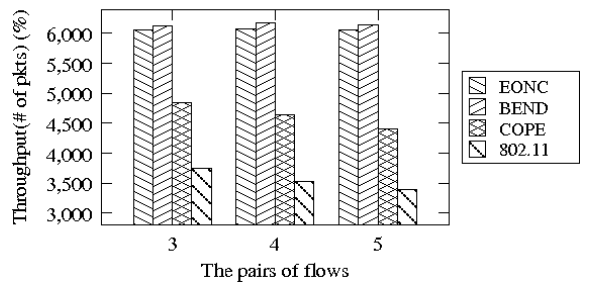

Fig. 2. Throughput of different schemes.

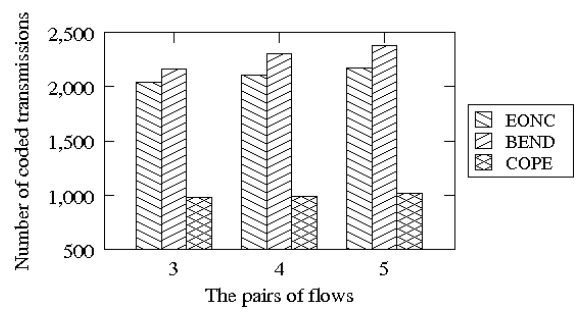

Fig. 3. Number of coded transmissions

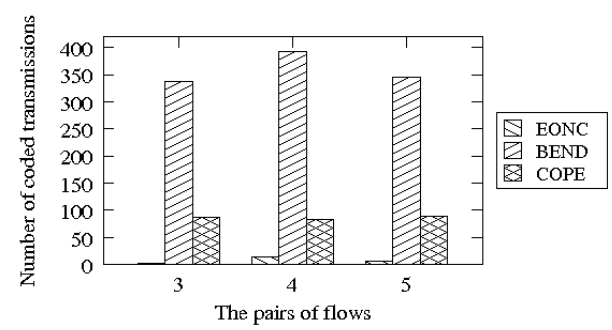

Fig. 4. Number of coded transmissions of the node with lowest residual energy.

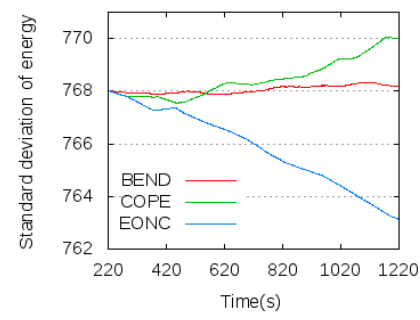

Fig. 5. Standard deviation of energy with four flow pairs.

Next, we evaluate the numbers of coded transmissions of the proposed network coding mechanism. As shown in Fig. 3, by introducing opportunistic forwarding, EONC and BEND achieves more coded transmissions, thus improving data transmission efficiency. Since we introduce energy metric into opportunistic forwarding, the number of coded transmissions decreases slightly, compared with BEND.

Fig. 4 provides the numbers of coded transmissions of the node with lowest residual energy. When performing EONC, the nodes with more residual energy will have more chances to forward the coded packets. More importantly, EONC selectively reduce opportunistic forwarding so as to prolong network lifetime.
Maximizing network lifetime is a major concern of WSNs. Figure 5 shows simulation on standard deviation of residual energy with the passage of time where we place four pairs of CBR flows. When EONC is performed, the lifetime of nodes with lower residual energy can be extended. The energy and congestion metrics contribute to achieve energy balance for WSNs, and therefore the lifetime of whole network will be extended.

\section{Conclusion}

In this paper, we propose the EONC to efficiently deliver data in heterogeneous WSNs, in consideration of congestion control. We begin with a dynamic classification approach in accordance in energy and congestion levels of the nodes that participant in opportunistic forwarding. Then, an adaptive opportunistic forwarding method is present to achieve energyefficient and congestion adaptive data delivery. Finally, we design a multi-priority data scheduling strategy, working together with the network coding mechanism, to ensure that packets have a better chance to be coded. Our simulation results demonstrate that, compared with typical network coding mechanisms COPE and BEND, the EONC achieves significant performance improvement in terms of energy efficiency and data transmission quality. According to this result, we determine that the proposed new techniques are feasible for WSNs.

Our ongoing work focuses on providing resiliency network coding opportunity when traffic congestion occurs.

\section{References}

[1] I. F. Akyildiz, W. Su, Y. Sankarasubramaniam and E. Cayirci, "A Survey on Sensor Networks," IEEE Communications Magazine, vol. 40, no. 8, pp. 102-116, August 2002.

[2] E. J. Duarte-Melo, M. Liu, "Analysis of energy consumption and lifetime of heterogeneous wireless sensor networks," in Proceedings of IEEE GLOBECOM, New York, NY, USA, pp. 21-25, March 2002.

[3] L. Qing, Q. Zhu, M. Wang, "Design of a distributed energy-efficient clustering algorithm for heterogeneous wireless sensor networks," Computer Communications, vol. 29, no. 12, pp. 2230-2237, August 2006.

[4] H. Shen, G. Bai, L. Zhao, and Z. Tang, "An Adaptive Opportunistic Network Coding Mechanism in Wireless Multimedia Sensor Networks," International Journal of Distributed Sensor Networks, vol. 2012, Article ID 565604, 13 pages, 2012.

[5] L. Miao, K. Djouani, A. Kurien, G. Noel, "Network coding and competitive approach for gradient based routing in wireless sensor networks," Ad Hoc Networks, vol. 10, no. 6, pp. 990-1008, 2012.

[6] Dan Wang, Qian Zhang, Jiangchuan Liu, "Partial Network Coding: Theory and Application for Continuous Sensor Data Collection," in Proceedings IWQoS, New Haven, CT, June, pp. 93-101, 2006.

[7] R. Ahlswede, N. Cai, S.-Y. R. Li, and R. W. Yeung, "Network information flow," IEEE Transactions on Information Theory, vol. 46, no. 4, pp. 1204-1216, 2000.

[8] S. Katti, H. Rahul, W. Hu, D. Katabi, M. Medard, and J. Crowcroft, "XORs in the air: practical wireless network coding," in Proceedings of ACM SIGCOMM, pp. 243-254, Pisa, Italy, 2006.

[9] J. Zhang, Y. P. Chen, and I. Marsic, "MAC-layer Proactive Mixing for Network Coding in Multi-hop Wireless Networks," Computer Networks, vol. 54, no. 2, pp. 196-207, 2010.

[10] G. Li, J. Li, B. Yu, "Lower bound of weighted fairness guaranteed congestion control protocol for wsns," in Proceedings of IEEE INFOCOM, Orlando, Florida, USA, pp. 3046-3050, March 2012.

[11] NS-2, http://nsnam.isi.edu/nsnam/index.php/Main_Page. 